\title{
Efficient Quantum Dot Light-Emitting Diodes Based on Trioctylphosphine Oxide-Passivated Organometallic Halide Perovskites
}

Yao Yao, ${ }^{\dagger}{ }^{\S}$ Hongtao $\mathrm{Yu},{ }^{\dagger, \S}$ Yanan $\mathrm{Wu},{ }^{\dagger}$ Yao Lu, ${ }^{\dagger}$ Ziwei Liu, ${ }^{+} \mathrm{Xin} \mathrm{Xu},{ }^{\dagger}$ Ben $\mathrm{Ma},{ }^{\dagger}$ Qing

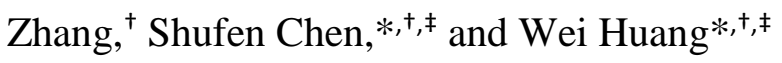

${ }^{\dagger}$ Key Laboratory for Organic Electronics and Information Displays \& Jiangsu Key Laboratory for Biosensors, Institute of Advanced Materials (IAM), Jiangsu National Synergetic Innovation Center for Advanced Materials (SICAM), Nanjing University of Posts \& Telecommunications, 9 Wenyuan Road, Nanjing 210023, China

${ }^{\ddagger}$ Institute of Flexible Electronics (SIFE), Northwestern Polytechnical University (NPU), 127 West Youyi Road, Xi'an 710072, Shaanxi, China

* Corresponding Author

E-mail address: iamsfchen@njupt.edu.cn (S. C.) and wei-huang@njtech.edu.cn (W. H.). 


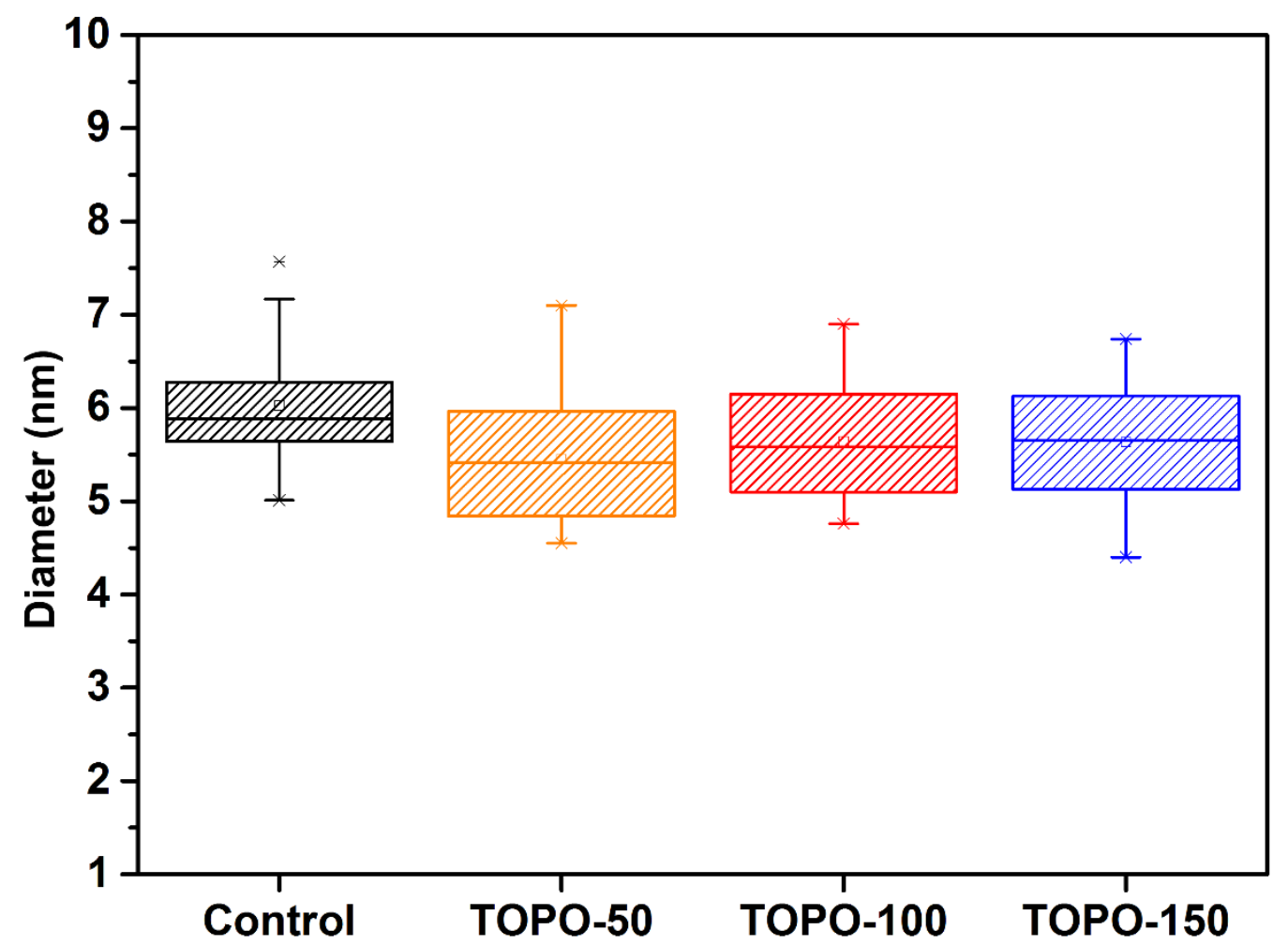

Figure S1. Statistical sizes of $\mathrm{CH}_{3} \mathrm{NH}_{3} \mathrm{PbBr}_{3}$ QDs with different TOPO concentrations. 


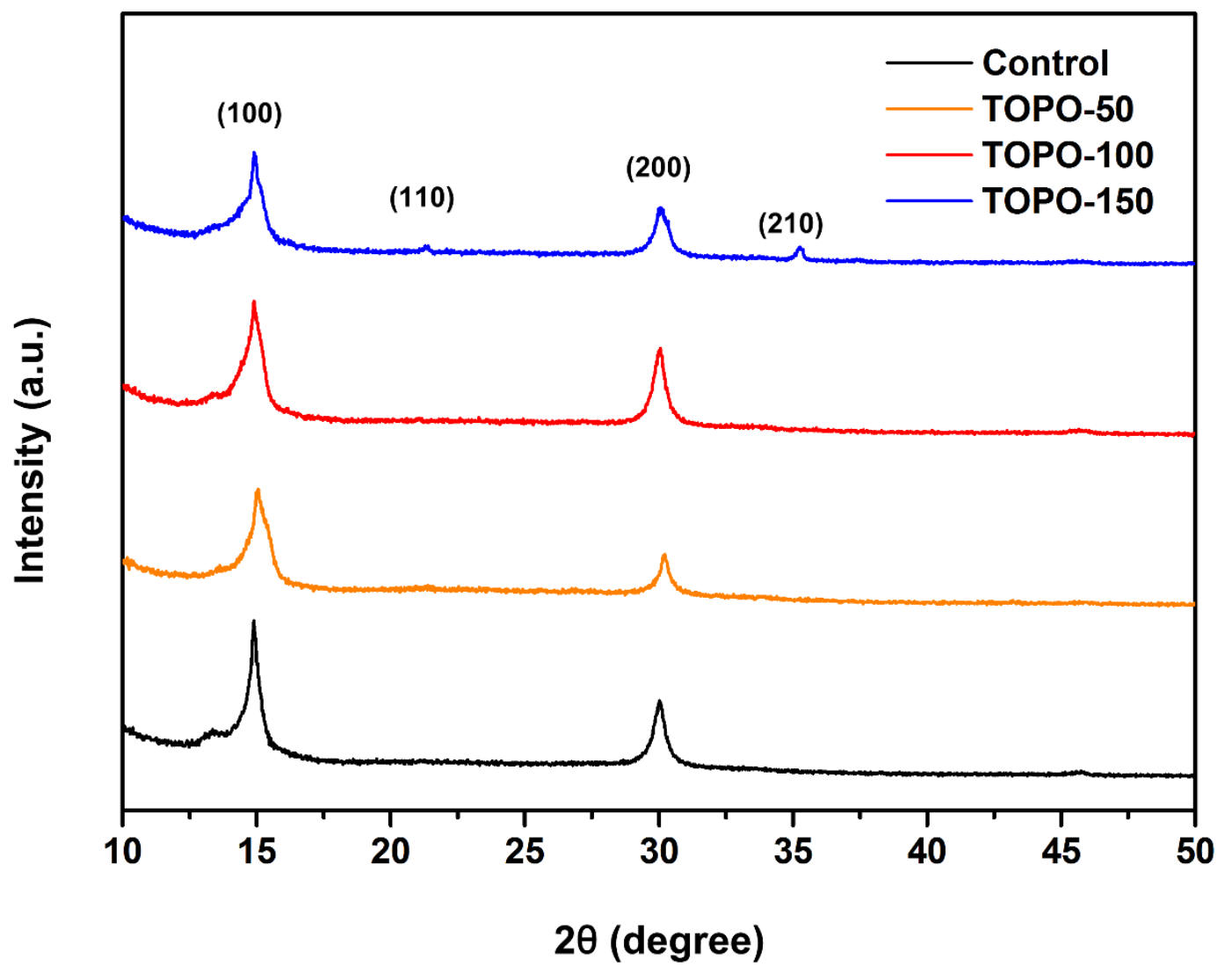

Figure S2. XRD patterns of samples at various TOPO concentrations. 
(a)

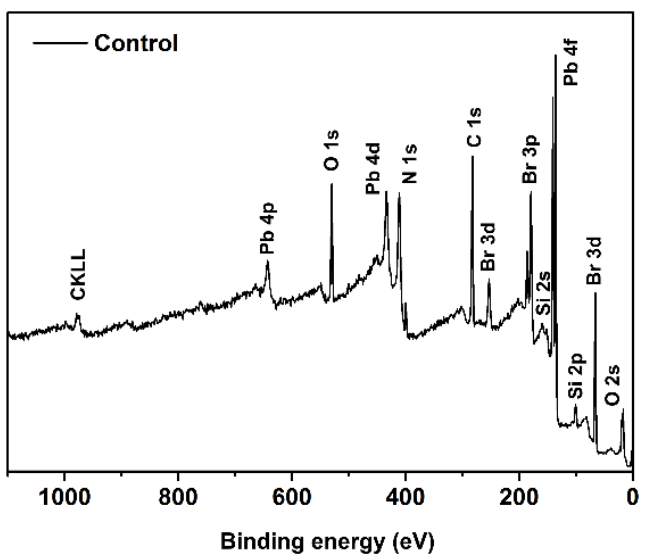

(c)

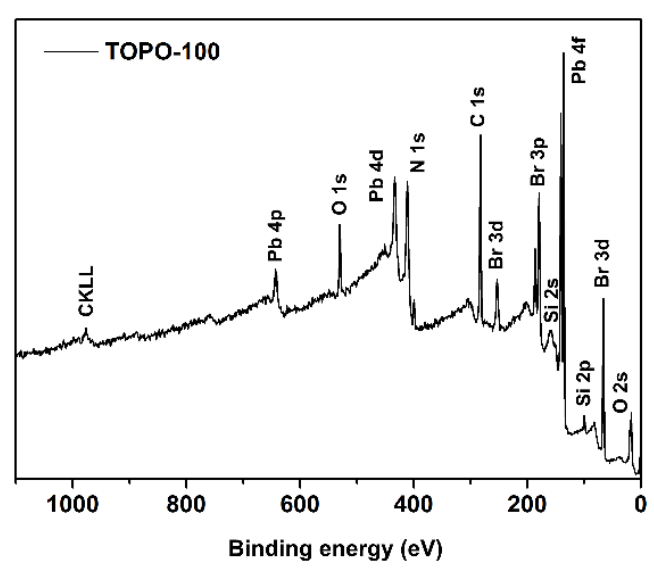

(b)

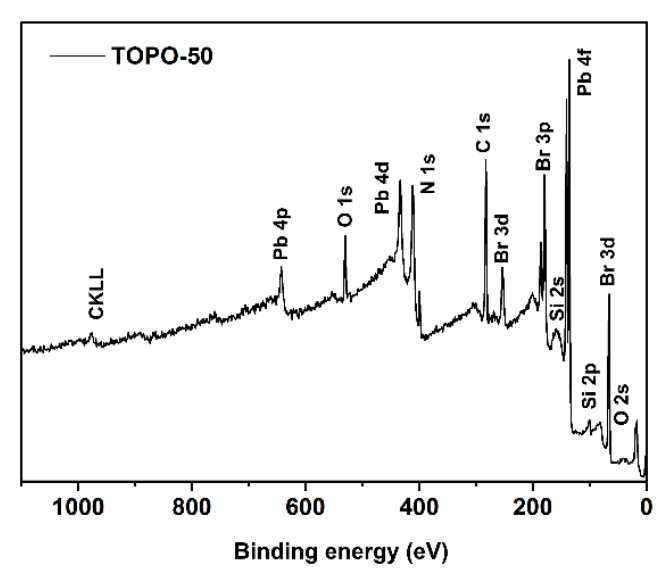

(d)

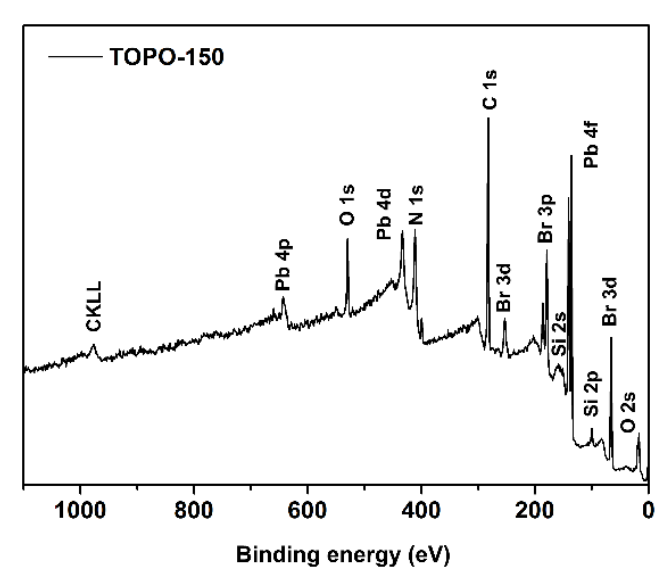

Figure S3. XPS survey scans of perovskite QDs of (a) Control, (b) TOPO-50, (c) TOPO-100, and (d) TOPO-150. 
$01 \mathrm{~s}$
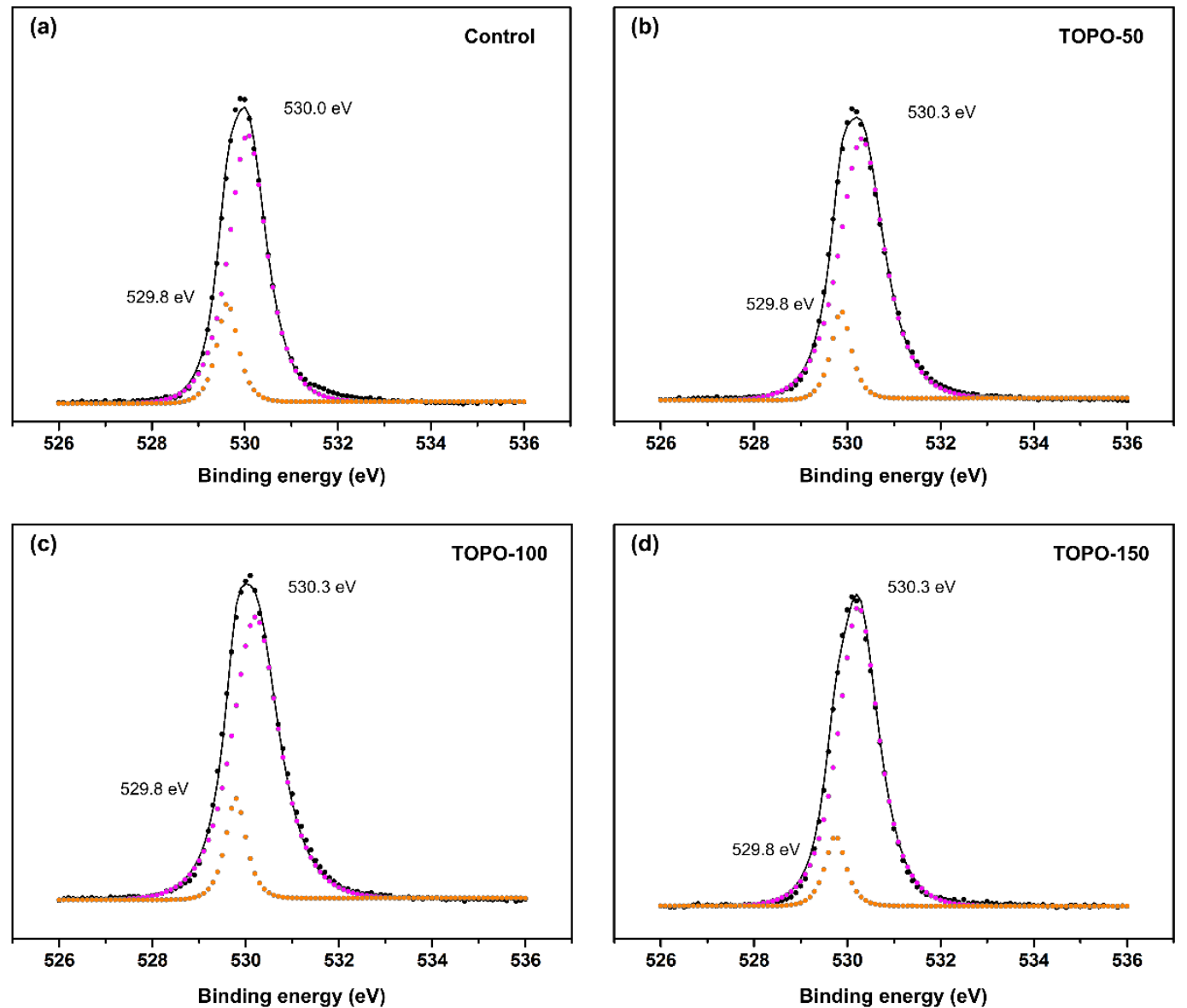

Figure S4. O 1s XPS spectra (black scatter-as measured; black solid line-the fitted data) of (a) Control, (b) TOPO-50, (c) TOPO-100, and (d) TOPO-150. 
$\operatorname{Br} 3 d$
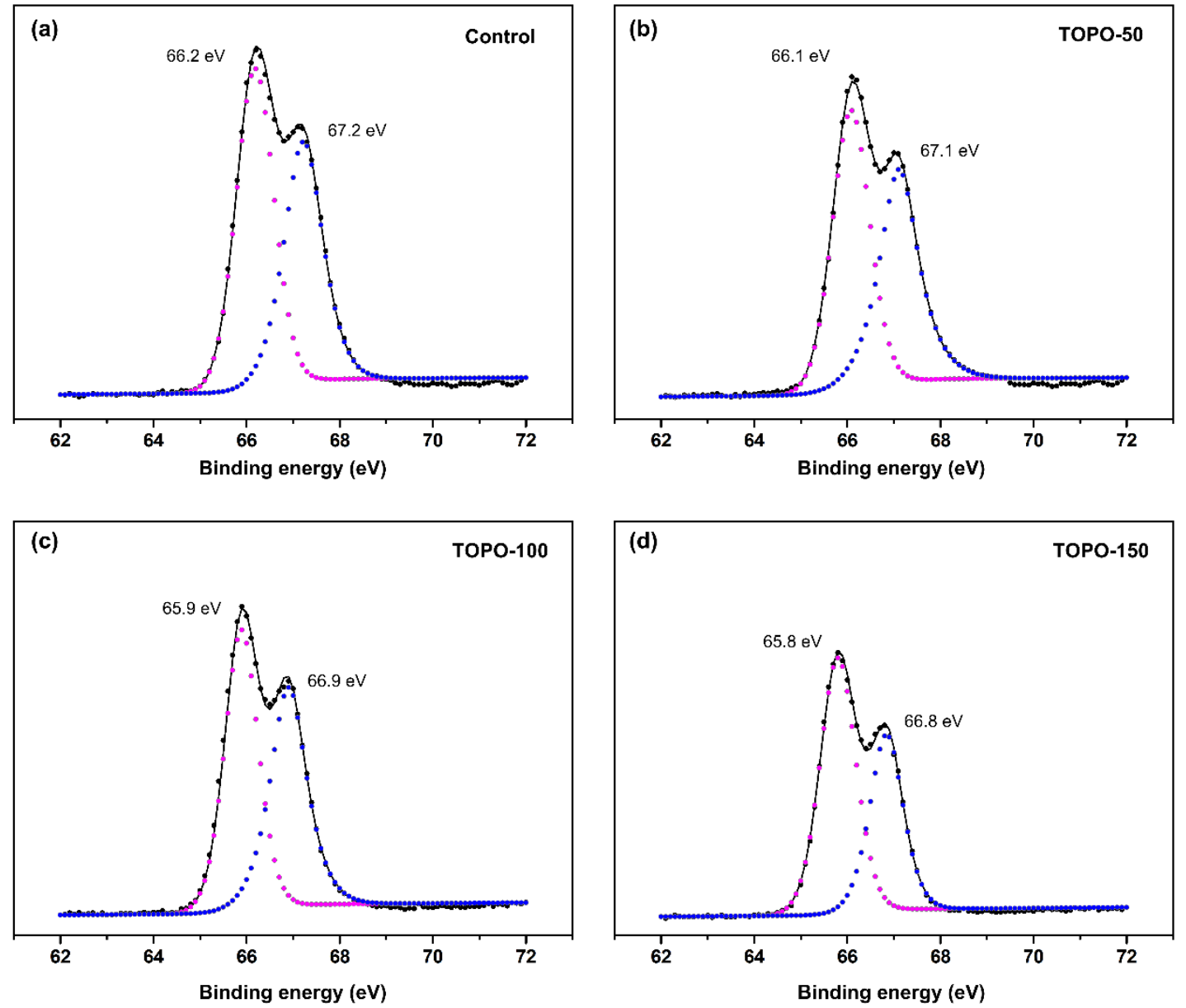

Figure S5. Br 3d XPS spectra (black scatter-as measured; black solid line-the fitted data) of (a) Control, (b) TOPO-50, (c) TOPO-100, and (d) TOPO-150. 

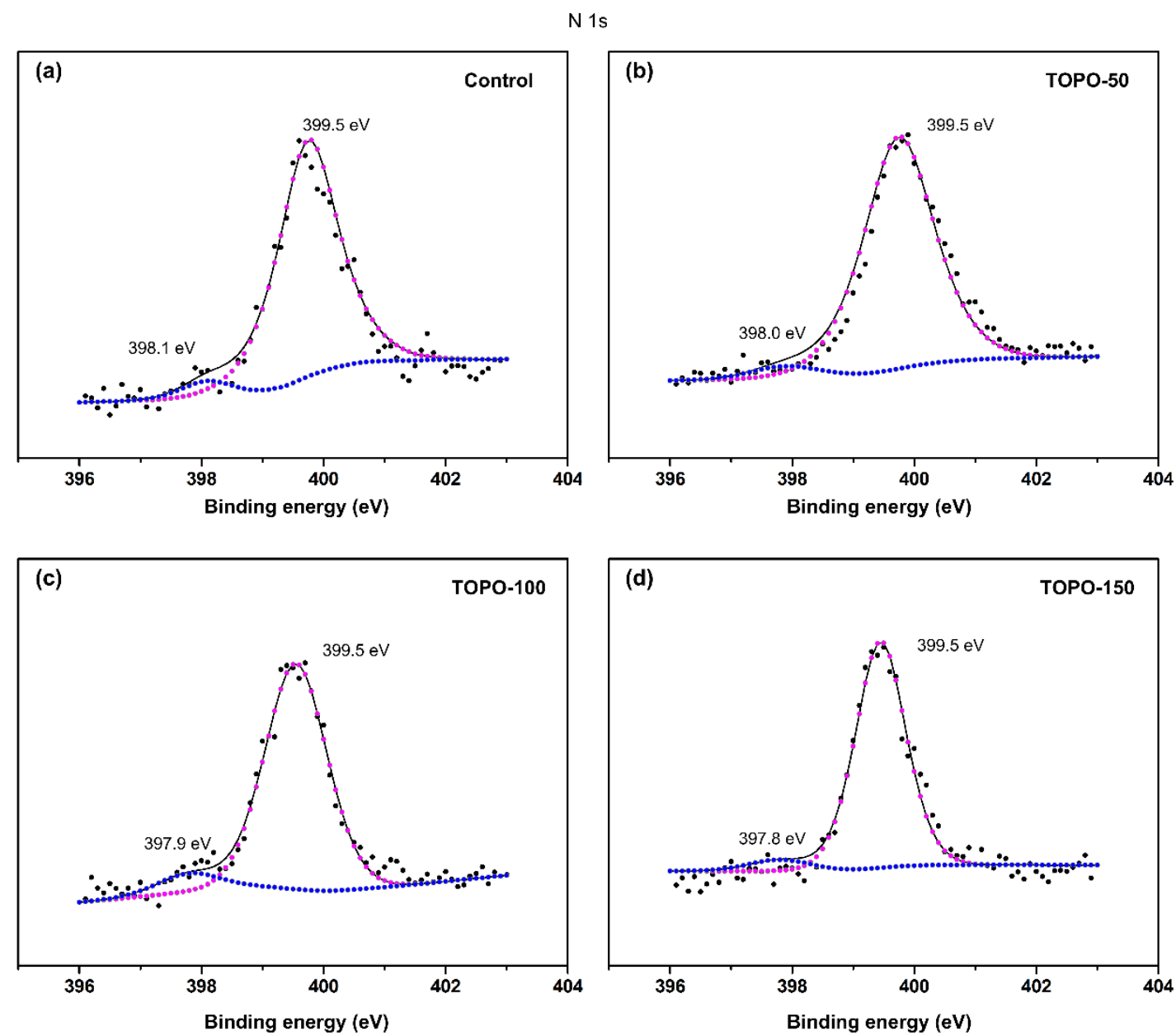

Figure S6. N 1s XPS spectra (black scatter-as measured; black solid line-the fitted data) of (a) Control, (b) TOPO-50, (c) TOPO-100, and (d) TOPO-150. 


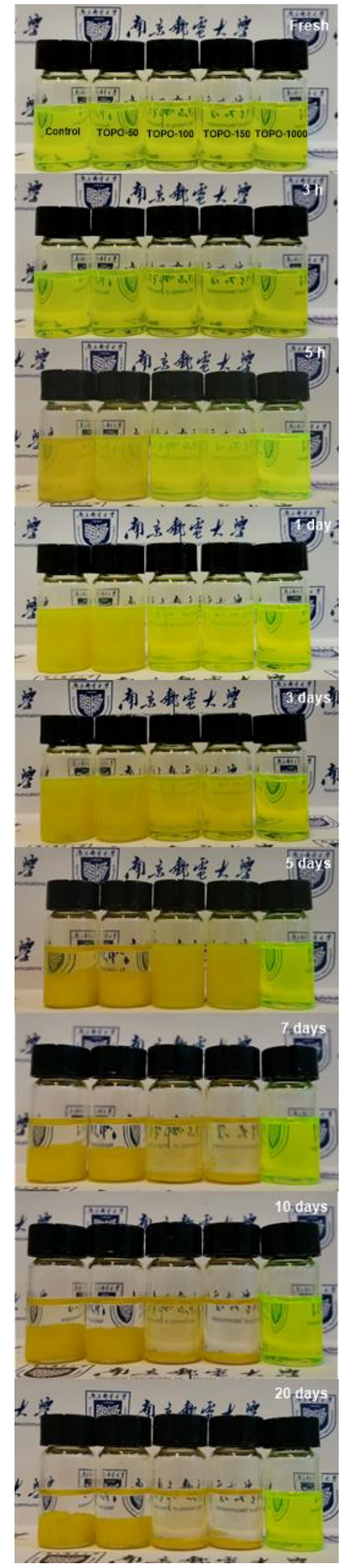

Figure S7. Solution stabilities of $\mathrm{CH}_{3} \mathrm{NH}_{3} \mathrm{PbBr}_{3}$ QDs with 0 (Control), 50 (TOPO-50), 100 (TOPO-100), $150 \mu \mathrm{L}$ (TOPO-150), and $1 \mathrm{~mL}$ (TOPO-1000) TOPO. 

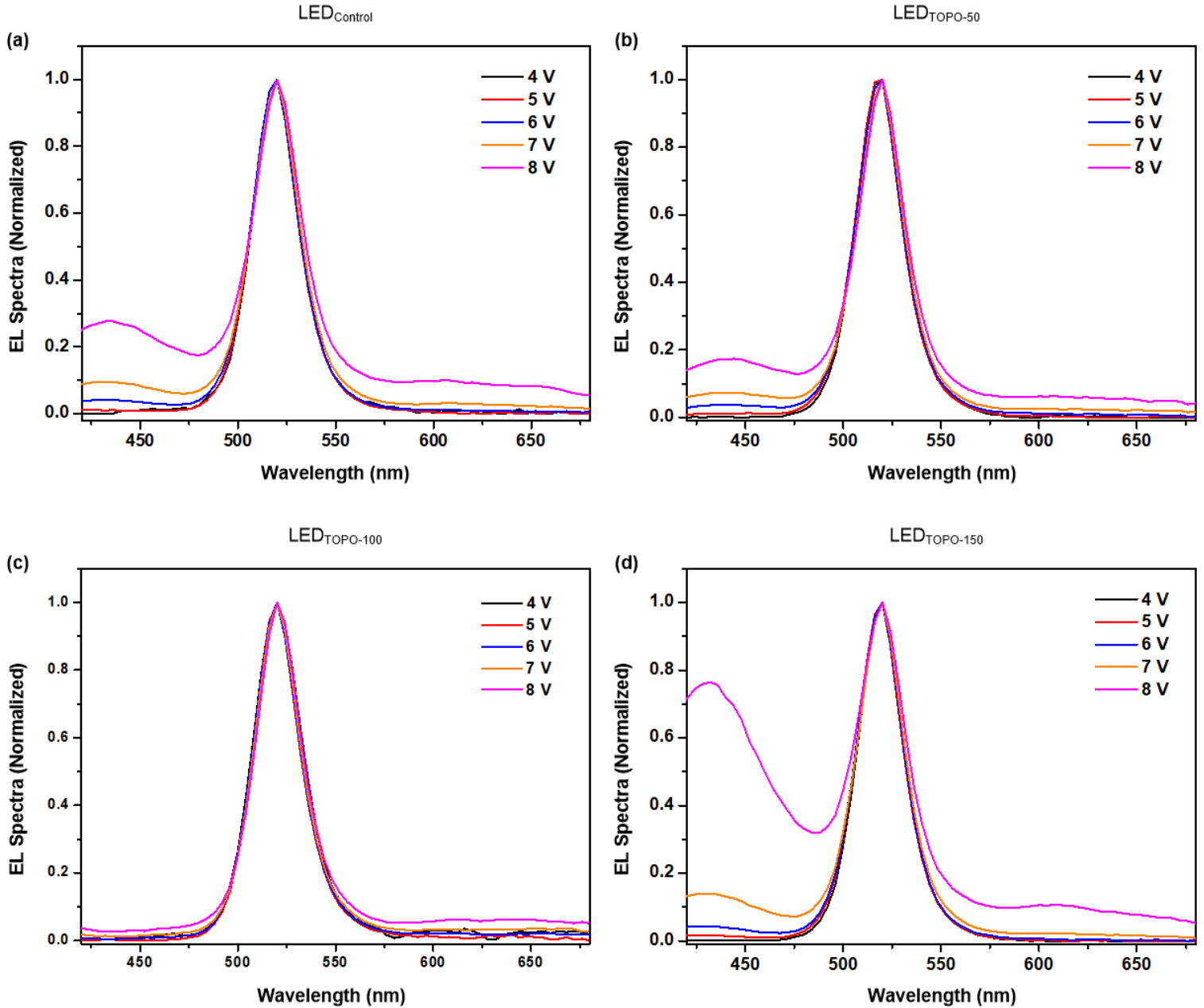

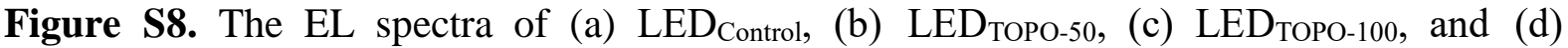
LED 


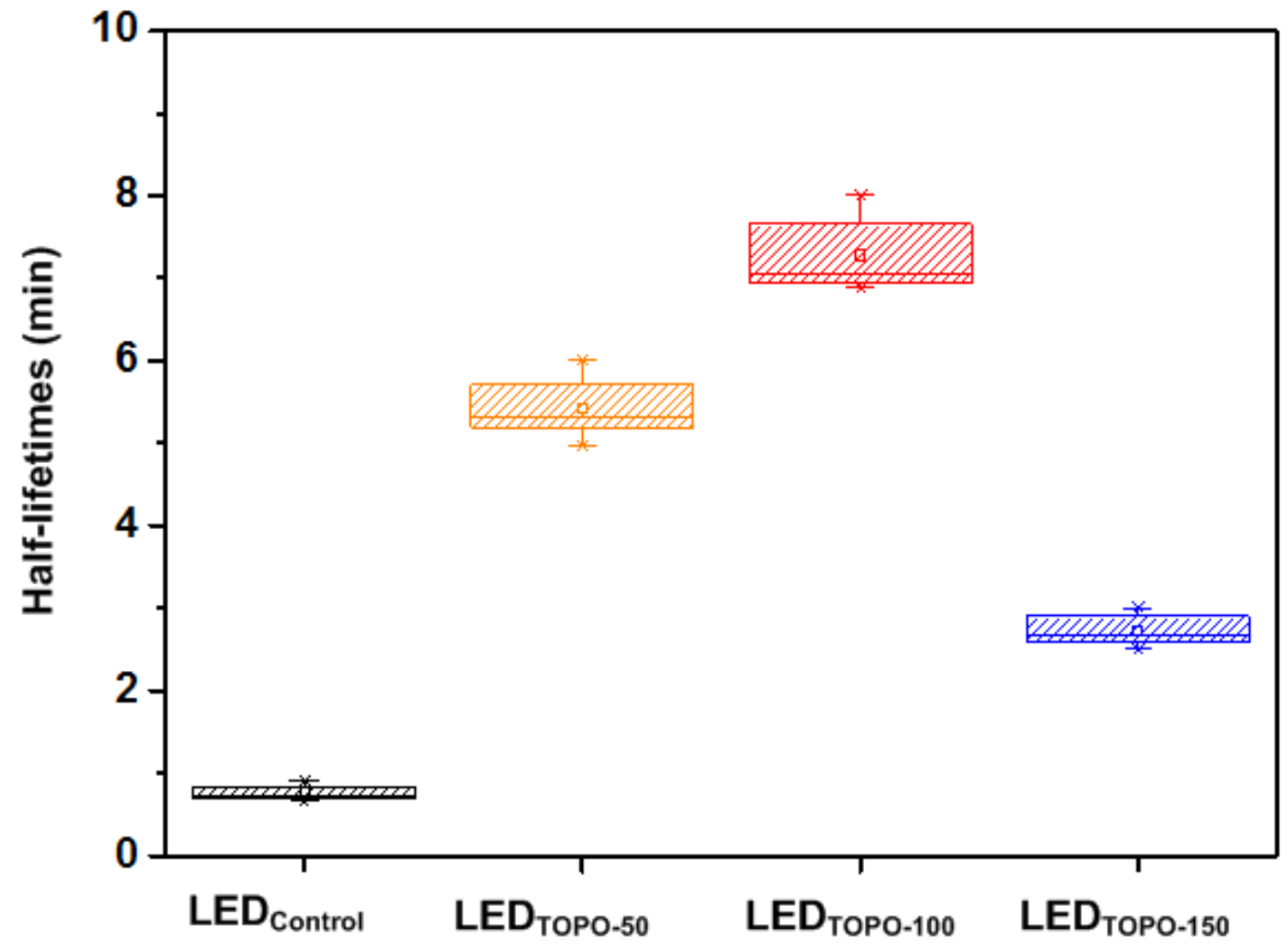

Figure S9. Half-lifetimes of LEDs with different TOPO concentrations 
Table S1. Summarized solution stabilities of perovskite QDs from previously published literatures and our work.

\begin{tabular}{|c|c|c|c|c|}
\hline QDs Types & PL Peak (nm) & PLQY (\%) & Lifetime & Ref. \\
\hline TOPO-passivated $\mathrm{MAPbBr}_{3}$ QDs & 520 & 69 & $>20$ days (50 RH\%) & This work \\
\hline L-cysteine-passivated $\mathrm{MAPbBr}_{3} \mathrm{NCs}$ & 533 & 53.7 & $2 \mathrm{~h}$ ( $73 \%$ of initial PL intensity) & 1 \\
\hline $\mathrm{MAPbBr}_{3} \mathbf{N P s}$ & 526 & 83 & $9 \mathrm{~h}$ ( $83 \%$ of initial PL intensity) & 2 \\
\hline $\mathrm{MAPbBr}_{3} \mathrm{NPs}$ & 527 & - & $>1$ day & 3 \\
\hline $\mathrm{MAPbBr}_{3} @ \mathrm{SiO}_{2} \mathrm{QDs}$ & 494 & 87 & 11 h (80 RH\%) & 4 \\
\hline $\mathrm{MAPbBr}_{3} \mathrm{NPs} / \mathrm{NaNO}_{3}$ & 525 & $\sim 100$ & after $30 \mathrm{~h}, 13.8 \%$ of initial PLQY & 5 \\
\hline $\mathrm{MAPbBr}_{3} @ \mathrm{SiO}_{2} \mathrm{QDs}$ & 520 & 77 & 3 days ( $94 \%$ of initial PL intensity) & 6 \\
\hline $\mathrm{MAPbBr}_{3} / \mathrm{MOF}-5$ QDs & 533 & 37.49 & 30 days (dispersed in water) & 7 \\
\hline $\mathrm{MAPbBr}_{3} / \mathrm{BnOH}$ & 525 & $95-99$ & $>4$ months (50 RH\%) & 8 \\
\hline $\mathrm{CspbBr}_{3} \mathrm{QDs}$ & 513 & 95 & $>30$ days & 9 \\
\hline $\mathrm{CsPbBr}_{3}$ QDs & 515 & $93 \pm 2.5$ & $>30$ days & 10 \\
\hline $\mathrm{CsPbBr}_{3} @ \mathrm{SiO}_{2} \mathrm{QDs}$ & 515 & 55 & $\begin{array}{l}4 \text { days }(80 \% \text { of initial PL intensity, under } \\
\text { continuous UV light) }\end{array}$ & 11 \\
\hline $\mathrm{CsPbBr}_{3} @$ POSS NCs & 514 & 69 & $>2$ months (dispersed in water) & 12 \\
\hline $\mathrm{CsPbBr}_{3} / \mathrm{SEBS}$ & 514 & $\sim 100$ & $>4$ months & 13 \\
\hline $\mathrm{CsPbBr}_{3} / \mathrm{DDAB}$ & 533 & 70 & $>4$ months $(60 \pm 5 \mathrm{RH} \%)$ & 14 \\
\hline
\end{tabular}


Table S2. Summarized device stabilities from some representative works. $\mathrm{T}_{50}$ and $\mathrm{T}_{70}$ are defined as the degradation time corresponding to $50 \%$ and $70 \%$ of the initial luminance $\left(\mathrm{L}_{0}\right)$, respectively.

\begin{tabular}{|c|c|c|c|c|c|}
\hline Emission layer & Device Structure & Ambient & Lifetime & $\begin{array}{c}\text { Max EQE } \\
(\%)\end{array}$ & Ref. \\
\hline $\mathrm{MAPbBr}_{3} \mathrm{QDs}$ & ITO/PEDOT:PSS/PVK/perovskite/TPBi/LiF/Al & air & $7 \min \left(T_{50}\right)$ & 1.64 & $\begin{array}{l}\text { This } \\
\text { work }\end{array}$ \\
\hline $\mathrm{MAPbBr}_{3} \mathbf{N P s}$ & ITO/PEDOT:PSS/perovskite/TPBi/TPBi: $\mathrm{Cs}_{2} \mathrm{CO}_{3} / \mathrm{Al}$ & - & $0.5 \min \left(\mathrm{T}_{50}\right)$ & 3.8 & 15 \\
\hline $\mathrm{MAPbBr}_{3} \mathrm{NCs}$ & ITO/PEDOT:PSS/perovskite/TPBi/LiF/Al & - & $1.7 \min \left(\mathrm{T}_{50}\right)$ & 8.21 & 16 \\
\hline $\begin{array}{c}\mathrm{MAPbBr} / \mathrm{BABr} \\
\text { quasi-core/shell NCs }\end{array}$ & ITO/PVK/perovskite/TPBi/LiF/Al & $\mathrm{N}_{2}$ & $1.5 \min \left(>\mathrm{L}_{0}\right)$ & 9.3 & 17 \\
\hline $\mathrm{MAPbBr}_{3} \mathrm{NCs}$ & $\begin{array}{l}\text { ITO/PEDOT:PSS/perovskite/B3PYMPM:TPBi/B3PYMPM:C } \\
\qquad \mathrm{s}_{2} \mathrm{CO}_{3} / \mathrm{Al}\end{array}$ & - & $6 \min \left(\mathrm{T}_{50}\right)$ & 12.9 & 18 \\
\hline $\mathrm{FAPbBr}_{3} \mathrm{NC}$ & ITO/PEDOT:PSS/TFB/FAPbBr 3 /TPBi/LiF/Al & air & $\sim 0.5 \min \left(\mathrm{T}_{50}\right)$ & 16.3 & 19 \\
\hline $\mathrm{CsPbBr}_{3} \mathrm{NCs}$ & $\mathrm{ITO} / \mathrm{ZnO} / \mathrm{CsPbBr}_{3} / \mathrm{TPBi} / \mathrm{Alq}_{3} / \mathrm{TPBi} / \mathrm{Cs}_{2} \mathrm{CO}_{3} / \mathrm{Al}$ & air & $7.7 \min \left(T_{50}\right)$ & 1.43 & 20 \\
\hline $\mathrm{CsPbBr}_{3} \mathrm{NC}$ & ITO/PEDOT:PSS/poly-TPD/CsPbBr $/ 3$ TPBi/ $\mathrm{Cs}_{2} \mathrm{CO}_{3} / \mathrm{Al}$ & air & $10.5 \min \left(\mathrm{T}_{50}\right)$ & 1.70 & 21 \\
\hline $\mathrm{CsPbBr}_{3} \mathrm{NC}$ & ITO/PEDOT:PSS/poly-TPD/PFI/CsPbBr $/ 3 / \mathrm{TPBi} / \mathrm{LiF} / \mathrm{Al}$ & $\mathrm{N}_{2}$ & $10 \min \left(\mathrm{T}_{50}\right)$ & 0.06 & 22 \\
\hline $\mathrm{CsPbBr}_{3} \mathrm{NCs}$ & ITO/PEDOT:PSS/perovskite/F8/Ca/Al & - & $9.5 \min \left(\mathrm{T}_{50}\right)$ & 0.008 & 23 \\
\hline $\begin{array}{l}\mathrm{CsPbBr}_{3} / \mathrm{MABr} \\
\text { quasi-core/shell }\end{array}$ & ITO/PEDOT:PSS/perovskite/B3PYMPM/LiF/Al & $\mathrm{N}_{2}$ & $10 \min \left(\mathrm{T}_{50}\right)$ & 20.3 & 24 \\
\hline silver-doped $\mathrm{CsPbI}_{3} \mathrm{NCs}$ & $\mathrm{Ag} / \mathrm{ZnO} / \mathrm{PEI} /$ perovskite/TCTA $/ \mathrm{MoO}_{3} / \mathrm{Au} / \mathrm{MoO}_{3}$ & air & $8 \min \left(T_{50}\right)$ & 11.2 & 25 \\
\hline silver-doped $\mathrm{CsPbI}_{3} \mathrm{NCs}$ & $\mathrm{Ag} / \mathrm{ZnO} / \mathrm{PEI} /$ perovskite/TCTA$/ \mathrm{MoO}_{3} / \mathrm{Au} / \mathrm{MoO}_{3}$ & $\mathrm{~N}_{2}$ & $40 \min \left(T_{50}\right)$ & 11.2 & 25 \\
\hline $\mathrm{CsPbBr}_{3} \mathrm{QDs}$ & $\mathrm{ITO} / \mathrm{NiO} / \mathrm{CsPBr}_{3} / \mathrm{ZnO} / \mathrm{Al}$ & $65 \mathrm{RH} \%$ & $1.75 \mathrm{~h}\left(\mathrm{~T}_{70}\right)$ & 0.11 & 26 \\
\hline $\mathrm{CsPbBr}_{3} \mathrm{NCs}$ & ITO/PEDOT:PSS/perovskite/TPBi/LiF/Al & $\begin{array}{l}\text { encapsula } \\
\text { tion }\end{array}$ & $70 \min \left(T_{50}\right)$ & 0.93 & 27 \\
\hline $\mathrm{Cs}_{0.87} \mathbf{M A}_{0.13} \mathrm{PbBr}_{3}$ & ITO/ZnO/PVP/perovskite/CBP/MoO $3 / \mathrm{Al}$ & - & $1 \mathrm{~h}\left(\approx \mathrm{L}_{0}\right)$ & 10.43 & 28 \\
\hline
\end{tabular}




\section{REFERENCES}

(1) Wang, S.; Zhou, L.; Huang, F.; Xin, Y.; Jin, P.; Ma, Q.; Pang, Q.; Chen, Y.; Zhang, J. Z. Hybrid organic-inorganic lead bromide perovskite supercrystals self-assembled with L-cysteine and their good luminescence properties. J. Mater. Chem. C 2018, 6 (41), 10994-11001.

(2) Gonzalez-Carrero, S.; Galian, R. E.; Pérez-Prieto, J. Maximizing the emissive properties of $\mathrm{CH}_{3} \mathrm{NH}_{3} \mathrm{PbBr}_{3}$ perovskite nanoparticles. J. Mater. Chem. A 2015, 3 (17), 9187-9193.

(3) Schmidt, L. C.; Pertegás, A.; González-Carrero, S.; Malinkiewicz, O.; Agouram, S.; Mínguez, E. G.; Bolink, H. J.; Galian, R. E.; Pérez-Prieto, J. Nontemplate synthesis of $\mathrm{CH}_{3} \mathrm{NH}_{3} \mathrm{PbBr}_{3}$ perovskite nanoparticles. J. Am. Chem. Soc. 2014, 136 (3), 850-853.

(4) Huang, S.; Li, Z.; Kong, L.; Zhu, N.; Shan, A.; Li, L. Enhancing the stability of $\mathrm{CH}_{3} \mathrm{NH}_{3} \mathrm{PbBr}_{3}$ quantum dots by embedding in silica spheres derived from tetramethyl orthosilicate in "waterless" toluene. J. Am. Chem. Soc. 2016, 138 (18), 5749-5752.

(5) Yang G.; Fan, Q.; Chen B.; Zhou, Q.; Zhong, H. Reprecipitation synthesis of luminescent $\mathrm{CH}_{3} \mathrm{NH}_{3} \mathrm{PbBr}_{3} / \mathrm{NaNO}_{3}$ nanocomposites with enhanced stability. J. Mater. Chem. C 2016, 4 (48), $11387-$ 11391.

(6) Liu, Z.; Zhang, Y.; Fan, Y.; Chen, Z.; Tang, Z.; Zhao, J.; Lv, Y.; Lin, J.; Guo, X.; Zhang, J.; Liu, X. Toward highly luminescent and stabilized silica coated perovskite quantum dots through simply mixing and stirring under room temperature in air. ACS Appl. Mater. Interfaces 2018, 10 (15), 13053-13061.

(7) Zhang, D.; Xu, Y.; Liu, Q.; Xia, Z. Encapsulation of $\mathrm{CH}_{3} \mathrm{NH}_{3} \mathrm{PbBr}_{3}$ perovskite quantum dots in MOF-5 microcrystals as a stable platform for temperature and qqueous heavy metal ion detection. Inorg. Chem. 2018, 57 (8), 4613-4619.

(8) Veldhuis, S. A.; Tay, Y. K. E.; Bruno, A.; Dintakurti, S. S.; Bhaumik, S.; Muduli, S. K.; Li, M.; Mathews, N.; Sum, T. C.; Mhaisalkar, S. G. Benzyl alcohol-treated $\mathrm{CH}_{3} \mathrm{NH}_{3} \mathrm{PbBr}_{3}$ nanocrystals exhibiting high luminescence, stability, and ultralow amplified spontaneous emission thresholds. Nano Lett. 2017, 17 (12), 7424-7432.

(9) Li, X.; Wu, Y.; Zhang, S.; Cai, B.; Gu, Y.; Song, J.; Zeng, H. CsPbX 3 quantum dots for lighting and displays: room-temperature synthesis, photoluminescence superiorities, underlying origins and white light-emitting diodes. Adv. Funct. Mater. 2016, 26 (15), 2435-2445.

(10) Song, J.; Li, J.; Xu, L.; Li, J.; Zhang, F.; Han, B.; Shan, Q.; Zeng, H. Room-temperature triple-ligand surface engineering synergistically boosts ink stability, recombination dynamics, and charge injection toward EQE-11.6\% perovskite QLEDs. Adv. Mater. 2018, 30 (30), 1800764.

(11) Wang, H. C.; Lin, S. Y.; Tang, A. C.; Singh, B. P.; Tong, H. C.; Chen, C. Y.; Lee, Y. C.; Tsai, T. L.; Liu, R. S. Mesoporous silica particles integrated with all-inorganic $\mathrm{CsPbBr}_{3}$ perovskite quantum-dot nanocomposites (MP-PQDs) with high stability and wide color gamut used for backlight display. Angew. Chem., Int. Ed. 2016, 55 (28), 7924-7929.

(12) Huang, H.; Chen, B.; Wang, Z.; Hung, T. F.; Susha, A. S.; Zhong, H.; Rogach, A. L. Water resistant $\mathrm{CsPX}_{3}$ nanocrystals coated with polyhedral oligomeric silsesquioxane and their use as solid state luminophores in all-perovskite white light-emitting devices. Chem. Sci. 2016, 7 (9), 5699-5703.

(13) Raja, S. N.; Bekenstein, Y.; Koc, M. A.; Fischer, S.; Zhang, D.; Lin, L.; Ritchie, R. O.; Yang, P.; Alivisatos, A. P. Encapsulation of perovskite nanocrystals into macroscale polymer matrices: enhanced stability and polarization. ACS Appl. Mater. Interfaces 2016, 8 (51), 35523-35533. 
(14) Pan, J.; Sarmah, S. P.; Murali, B.; Dursun, I.; Peng, W.; Parida, M. R.; Liu, J.; Sinatra, L.; Alyami, N.; Zhao, C.; Alarousu, E.; Ng, T. K.; Ooi, B. S.; Bakr, O. M.; Mohammed, O. F. Air-stable surface-passivated perovskite quantum dots for ultra-robust, single- and two-photon-induced amplified spontaneous emission. J. Phys. Chem. Lett. 2015, 6 (24), 5027-5033.

(15) Xing, J.; Yan, F.; Zhao, Y.; Chen, S.; Yu, H.; Zhang, Q.; Zeng, R.; Demir, H. V.; Sun, X.; Huan, A.; Xiong, Q. High-efficiency light-emitting diodes of organometal halide perovskite amorphous nanoparticles. ACS Nano 2016, 10 (7), 6623-6630.

(16) Lee, J.-W.; Choi, Y. J.; Yang, J.-M.; Ham, S.; Jeon, S. K.; Lee, J. Y.; Song, Y.-H.; Ji, E. K.; Yoon, D.-H.; Seo, S.; Shin, H.; Han, G. S.; Jung, H. S.; Kim, D.; Park, N.-G. In-situ formed type I nanocrystalline perovskite film for highly efficient light-emitting diode. ACS Nano 2017, 11 (3), 3311-3319.

(17) Xiao, Z.; Kerner, R. A.; Zhao, L.; Tran, N. L.; Lee, K. M.; Koh, T.-W.; Scholes, G. D.; Rand, B. P. Efficient perovskite light-emitting diodes featuring nanometre-sized crystallites. Nat. Photonics 2017, 11, 108.

(18) Yan, F.; Xing, J.; Xing, G.; Quan, L.; Tan, S. T.; Zhao, J.; Su, R.; Zhang, L.; Chen, S.; Zhao, Y.; Huan, A.; Sargent, E. H.; Xiong, Q.; Demir, H. V. Highly efficient visible colloidal lead-halide perovskite nanocrystal light-emitting diodes. Nano Lett. 2018, 18 (5), 3157-3164.

(19) Han, D.; Imran, M.; Zhang, M.; Chang, S.; Wu, X.-g.; Zhang, X.; Tang, J.; Wang, M.; Ali, S.; Li, X. Efficient light-emitting diodes based on in situ fabricated $\mathrm{FAPbBr}_{3}$ nanocrystals: the enhancing role of the ligand-assisted reprecipitation process. ACS Nano 2018, 12 (8), 8808-8816.

(20) Liu, B.; Wang, L.; Gu, H.; Sun, H.; Demir, H. V. Highly efficient green light-emitting diodes from all-inorganic perovskite nanocrystals enabled by a new electron transport layer. Adv. Opt. Mater. 2018, 6 (11), 1800220.

(21) Wang, L.; Liu, B.; Zhao, X.; Demir, H. V.; Gu, H.; Sun, H. Solvent-assisted surface engineering for high-performance all-inorganic perovskite nanocrystal light-emitting diodes. ACS Appl. Mater. Interfaces 2018, 10 (23), 19828-19835.

(22) Zhang, X.; Lin, H.; Huang, H.; Reckmeier, C.; Zhang, Y.; Choy, W. C.; Rogach, A. L. Enhancing the brightness of cesium lead halide perovskite nanocrystal based green light-emitting devices through the interface engineering with perfluorinated ionomer. Nano Lett. 2016, 16 (2), 1415-1420.

(23) Yantara, N.; Bhaumik, S.; Yan, F.; Sabba, D.; Dewi, H. A.; Mathews, N.; Boix, P. P.; Demir, H. V.; Mhaisalkar, S. Inorganic halide perovskites for efficient light-emitting diodes. J. Phys. Chem. Lett. 2015, 6 (21), 4360-4364.

(24) Lin, K.; Xing, J.; Quan, L. N.; de Arquer, F. P. G.; Gong, X.; Lu, J.; Xie, L.; Zhao, W.; Zhang, D.; Yan, C. Perovskite light-emitting diodes with external quantum efficiency exceeding 20 percent. Nature 2018, 562 (7726), 245.

(25) Lu, M.; Zhang, X.; Bai, X.; Wu, H.; Shen, X.; Zhang, Y.; Zhang, W.; Zheng, W.; Song, H.; Yu, W. W. Spontaneous silver doping and surface passivation of $\mathrm{CsPbI}_{3}$ perovskite active layer enable light-emitting devices with an external quantum efficiency of 11.2\%. ACS Energy Lett. 2018, 3 (7), 1571-1577.

(26) Shan, Q.; Li, J.; Song, J.; Zou, Y.; Xu, L.; Xue, J.; Dong, Y.; Huo, C.; Chen, J.; Han, B. All-inorganic quantum-dot light-emitting diodes based on perovskite emitters with low turn-on voltage and high humidity stability. J. Mater. Chem. C 2017, 5 (18), 4565-4570.

(27) Zhang, X.; Wang, W.; Bing, X.; Sheng, L.; Dai, H.; Bian, D.; Chen, S.; Kai, W.; Xiao, W. S. Thin film perovskite light-emitting diode based on $\mathrm{CsPbr}_{3}$ powders and interfacial engineering. Nano Energy 2017, 
$37,40-45$.

(28) Zhang, L.; Yang, X.; Jiang, Q.; Wang, P.; Yin, Z.; Zhang, X.; Tan, H.; Yang, Y. M.; Wei, M.; Sutherland, B. R. Ultra-bright and highly efficient inorganic based perovskite light-emitting diodes. Nat. Commun. 2017, 8, 15640. 